\title{
Impatto della pandemia da COVID-19 sullo stress psicofisico nei pazienti con insufficienza surrenalica
}

\author{
Federica Spagnolo ${ }^{1}$. Francesco Trimarchi ${ }^{2}$
}

Accettato: 29 dicembre 2020 / Pubblicato online: 14 maggio 2021

(c) The Author(s), under exclusive licence to Springer Nature Switzerland AG 2021

\section{Commento a:}

Impact of COVID19 pandemic on psychophysical stress in patients with adrenal insufficiency:

the CORTICOVID study.

M. Martino, N. Aboud, M.F. Cola, G. Giancola,

A. Ciarloni, G. Salvio, G. Arnaldi.

J Endocrinol Invest (2020) 18:1-10

Fra i pazienti con endocrinopatie croniche, quelli affetti da insufficienza surrenalica (AI) sono sicuramente fra i più vulnerabili, non solo per un'aumentata suscettibilità alle infezioni, secondaria alla nota compromissione del sistema immunitario [1], ma anche e soprattutto per il rischio di intercorrenti crisi surrenaliche che possono complicare il decorso di tali infezioni così come di altri eventi "stressanti" per il paziente $[2,3]$.

Per questi e altri motivi, la pandemia da SARS-COV-2 rappresenta un fattore imprevisto che influenza pesantemente nei pazienti con AI la gestione complessiva della terapia sostitutiva con glucocorticoidi $[4,5]$. Il rischio che si configura, infatti, oltre a quello di un'infezione virale con possibile evoluzione severo-critica in circa il $20 \%$ dei casi [6] è quello di un evento straordinario che, impattando così fortemente sui diversi aspetti della vita quotidiana, porta necessariamente con sé un carico psicologico ed emotivo a volte imprevedibile [7]. Si aggiunga poi, che la cosiddetta mano invisibile [8] ha dato una forte spinta all'endocrinologia clinica verso un futuro inatteso e condizionato dalla necessità di garantire assistenza domiciliare a distanza attraverso

$\triangle$ F. Trimarchi

francesco.trimarchi@unime.it

1 Distretto Sanitario di Cividale e Tarcento, Azienda Sanitaria Universitaria Friuli Centrale, Udine, Italia

2 Dipartimento di Medicina clinica e sperimentale, Università degli Studi di Messina, Messina, Italia l'utilizzo di nuove tecnologie, ormai riconosciute e normate sotto il termine di telemedicina.

In tale contesto, questo studio monocentrico condotto tra febbraio e aprile 2020, durante l'esplosione della pandemia e il confinamento della popolazione italiana, rappresenta il primo e l'unico disegnato per valutare in questi pazienti la prevalenza, le manifestazioni cliniche e l'esito della malattia COVID-19, la prevalenza di crisi surrenaliche e la loro associazione con l'infezione da SARS-COV2 e/o con lo stress psicofisico ad essa correlato nonché l'impatto emotivo dell'isolamento e del distanziamento sociale in tale circostanza. A questo scopo è stato somministrato per e-mail o telefonicamente a 121 pazienti $(59 \mathrm{M}, 40$ AI primitiva e 81 AI secondaria) un questionario appositamente progettato e denominato CORTI-COVID, costituito da 34 domande facenti riferimento a 4 ambiti di indagine. Il primo relativo ai dati demografici e antropometrici del paziente, il secondo all'anamnesi patologica e farmacologica generale e AIspecifica, il terzo relativo alla storia familiare, fisiologica e medica conseguente all'infezione da SARS-COV2, il quarto all'impatto emotivo della pandemia. Proprio da quest'ultimo dipende il CORTI-COVID Score calcolato su 5 domande inerenti al grado crescente (da 1 a 5) di preoccupazione per la salute globale, quella personale in quanto paziente con AI, le conseguenze occupazionali, le implicazioni economiche e infine sociali della pandemia. Al fine di validare e supportare l'affidabilità di tale questionario, gli autori hanno utilizzato l'AddQoL-30 [9-11] e la Short-form-36-Health Survey (SF 36) [12], due dei questionari più ampiamente riconosciuti per l'auto-valutazione della qualità di vita in pazienti affetti da AI primaria e nella popolazione generale, rispettivamente.

Per quanto riguarda alcuni dei principali endpoints dello studio, è necessario osservare che, nonostante nel $23 \%$ dei casi venga riferita nei contatti stretti una storia di positività a 
SARS-COV2, solo una paziente di 48 anni con AI primaria sviluppa l'infezione ( 0,8 vs $2,5 \%$ della popolazione italiana nello stesso periodo) senza necessità di ospedalizzazione, e in nessuno dei pazienti viene segnalata l'insorgenza di crisi surrenaliche. Tuttavia, ciò che emerge dalla valutazione dell'impatto emotivo della pandemia è un CORTI-COVID Score con un valore medio piuttosto alto $(17,7)$ senza differenze tra AI primaria e secondaria, né differenze significative tra chi mantiene la stessa terapia sostitutiva con glucocorticoidi e chi, invece, ricorre a un adeguamento della dose (6 AI primaria, 8 AI secondaria), in 6/14 casi (5 donne con AI primaria, 1 uomo con AI secondaria) attribuito al maggiore stress psico-emotivo. Questi ultimi, rispetto agli altri pazienti, si presentano del tutto simili per età, sesso, eziologia della AI, dose sostitutiva di glucocorticoidi e comorbidità, eccetto che per la maggiore prevalenza di malattie autoimmuni. Inoltre, i pazienti affetti da concomitanti patologie autoimmuni mostrano una più alta preoccupazione per la salute personale, che è quella che maggiormente impatta lo score finale dei pazienti con AI primaria, nei quali le stesse hanno una maggiore prevalenza. Diversamente, nei pazienti con AI secondaria la componente più rilevante nel calcolo del CORTI-COVID Score è data dalla preoccupazione per le conseguenze economiche della pandemia, a sua volta correlata in maniera inversa con l'età. Ma anche nei pazienti con AI secondaria la presenza di comorbidità assume un certo ruolo, laddove l'ipertensione arteriosa si associa a un maggior timore per la salute globale e le malattie autoimmuni per la salute personale.

$\mathrm{Nel}$ caso del questionario ADDIQoL-30, invece, si evidenzia una migliore qualità di vita nel sesso maschile e in pazienti che non presentano storia di diabete e cardiopatia ischemica rispetto a tutti gli altri. Risultati che vengono sostanzialmente ripresi e confermati dalla FS-36 survey. I due questionari, infatti, presentano una significativa correlazione diretta tra loro, in particolare per quanto riguarda la percezione di "astenia" e "benessere emotivo", e inversa con il CORTI-COVID Score. Correlazioni che vengono confermate anche dividendo la popolazione in AI primaria e secondaria.

Contrariamente dunque alle nostre premesse, degna di nota in questo studio è sicuramente l'evidenza che i pazienti affetti da AI non presentano un tasso d'infezione da SARSCOV2 superiore alla popolazione generale, né una maggiore suscettibilità a crisi surrenaliche conseguenti all'impatto emotivo e psico-sociale della pandemia. Rilevante anche il fatto che, malgrado valori di CORTI-COVID Score diffusamente alti, solo il 5\% circa della popolazione in studio ha dovuto adeguare la terapia sostitutiva con glucocorticoide per sopperire all'aumentato stress psico-fisico. Ciononostante, il dato che quest'ultimi, seppure con numeri ristrettissimi, siano per la quasi totalità donne affette da AI primaria, che in caso di AI primaria il CORTI-COVID Score risulti significativamente impattato dall'apprensione per la salute personale e che questa si associ a una maggiore prevalenza di malattie autoimmuni (in entrambi i gruppi), assieme con quanto emerso dai questionari ADDIQoL-30 e SF-36, suggerisce una minore capacità di adattamento allo stress "psico-emotivo" in pazienti con forma primaria, con malattie autoimmuni concomitanti e/o di sesso femminile. Mentre i pazienti affetti da AI secondaria sembrerebbero meno consapevoli della loro condizione di fragilità concentrandosi sull'impatto economico della pandemia o, viceversa, sulla salute globale in generale quando affetti da altre comorbidità, come l'ipertensione, note per peggiorare la prognosi della malattia COVID-19.

Pertanto, particolarmente interessante sarebbe non solo verificare questi primi risultati su una popolazione più ampia, magari con uno studio multicentrico, ma anche confrontare i risultati ottenuti dai pazienti con AI con quelli di un gruppo di controllo.

In ultimo, e non meno importante, è il ruolo innovativo che tale "esperimento" rappresenta in simile contesto, introducendo uno strumento che, facilmente adattabile ad altre situazioni, risulta utile alla "misurazione" dello stato di stress del paziente. Questo, diventando quantificabile per l'endocrinologo, permette un adeguamento della terapia sostitutiva tempestivo e consapevole così da prevenire sia crisi surrenaliche estremamente pericolose, sia eventuali trattamenti eccessivi fai-da-te. Non si può escludere, infatti, che i risultati dello studio [13] siano anche frutto dell'influenza positiva che tale indagine osservazionale ha avuto sul paziente, in virtù della percezione di "intervento" resa rispetto alla condizione di isolamento e abbandono relativa alla pandemia.

Nota della casa editrice Springer Nature rimane neutrale in riguardo alle rivendicazioni giurisdizionali nelle mappe pubblicate e nelle affiliazioni istituzionali.

\section{Bibliografia}

1. Bancos I, Hazeldine J, Chortis V et al (2017) Primary adrenal insufficiency is associated with impaired natural killer cell function: a potential link to increased mortality. Eur $\mathrm{J}$ Endocrinol 176(4):471-480

2. Smans LC, Van der Valk ES, Hermus AR, Zelissen PM (2016) Incidence of adrenal crisis in patients with adrenal insufficiency. Clin Endocrinol (Oxf) 84(1):17-22

3. Hahner S, Spinnler C, Fassnacht $M$ et al (2015) High incidence of adrenal crisis in educated patients with chronic adrenal insufficiency: a prospective study. J Clin Endocrinol Metab 100(2):407-416

4. Isidori AM, Arnaldi G, Boscaro M et al (2020) COVID-19 infection and glucocorticoids: update from the Italian Society of Endocrinology expert opinion on steroid replacement in adrenal insufficiency. J Endocrinol Invest 43(8):1141-1147

5. Arlt W, Baldeweg SE, Pearce SHS, Simpson HL (2020) Endocrinology in the time of COVID-19: management of adrenal insufficiency. Eur J Endocrinol 183(1):G25-G32 
6. Wu Z, McGoogan JM (2020) Characteristics of and important lessons from the Coronavirus disease 2019 (COVID-19) outbreak in China: summary of a report of 72314 cases from the Chinese Center for Disease Control and Prevention. JAMA 323(13):1239-1242

7. Sani G, Janiri D, Di Nicola M et al (2020) Mental health during and after the COVID-19 emergency in Italy. Psychiatry Clin Neurosci 74(6):372

8. Trimarchi F (2020) An invisible hand has guided clinical endocrinology toward an unexpected future. J Endocrinol Invest 43:1037-1038

9. Løvås K, Curran S, Oksnes M et al (2010) Development of a disease-specific quality of life questionnaire in Addison's disease. J Clin Endocrinol Metab 95(2):545-551
10. Øksnes M, Bensing S, Hulting AL et al (2012) Quality of life in European patients with Addison's disease: validity of the disease-specific questionnaire AddiQoL. J Clin Endocrinol Metab 97(2):568-576

11. Meyer G, Koch M, Herrmann E et al (2018) Longitudinal AddiQoL scores may identify higher risk for adrenal crises in Addison's disease. Endocrine 60(2):355-361

12. Ware JE Jr, Sherbourne CD (1992) The MOS 36-item shortform health survey (SF-36). I. Conceptual framework and item selection. Med Care 30(6):473-483

13. Martino M, Aboud N, Cola MF et al (2020) Impact of COVID19 pandemic on psychophysical stress in patients with adrenal insufficiency: the CORTICOVID study. J Endocrinol Invest 18:1-10 